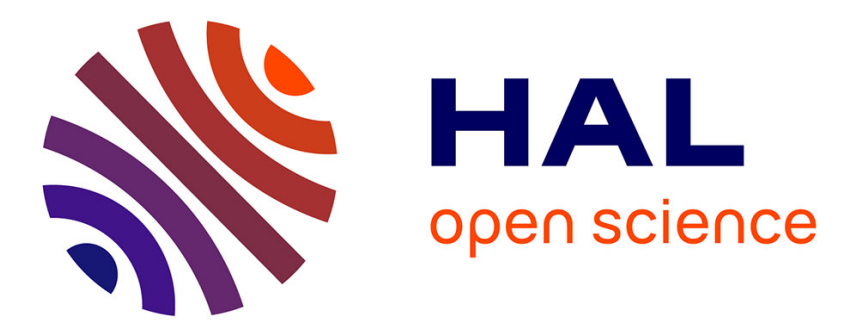

\title{
Decentralized and autonomous design for FDI/FTC of networked control systems
}

Dominique Sauter, Taha Boukhobza, Frédéric Hamelin

\section{To cite this version:}

Dominique Sauter, Taha Boukhobza, Frédéric Hamelin. Decentralized and autonomous design for FDI/FTC of networked control systems. Aug 2006, pp.CDROM. hal-00120858

\section{HAL Id: hal-00120858 \\ https://hal.science/hal-00120858}

Submitted on 18 Dec 2006

HAL is a multi-disciplinary open access archive for the deposit and dissemination of scientific research documents, whether they are published or not. The documents may come from teaching and research institutions in France or abroad, or from public or private research centers.
L'archive ouverte pluridisciplinaire HAL, est destinée au dépôt et à la diffusion de documents scientifiques de niveau recherche, publiés ou non, émanant des établissements d'enseignement et de recherche français ou étrangers, des laboratoires publics ou privés. 


\title{
DECENTRALIZED AND AUTONOMOUS DESIGN FOR FDI/FTC OF NETWORKED CONTROL SYSTEMS
}

\author{
Dominique Sauter, Taha Boukhobza and Frédéric Hamelin
}

\author{
Centre de Recherche en Automatique de Nancy (CRAN), \\ Nancy-University, CNRS UMR 7039 \\ BP 239, 54506 Vandouvre Cedex, Nancy, France, \\ E-mail: dominique.sauter@cran.uhp-nancy.fr
}

\begin{abstract}
In this paper, the fault tolerant control problem is addressed in a networked framework. An isolation filter together with a fault compensation mechanism are proposed for FDI/FTC. Several design procedures are studied. First, in the centralized architecture, the inputs and outputs information used for FDI \& FTC are collected and processed in a central node. Considering that sub-systems exchange information over the network, a decentralized architecture is then proposed for FDI/FTC. The autonomous capability of the decentralized design is also considered. But, whatever the design architecture is, fault detectability and isolability, are dependant on the available information. A method based on system structural analysis is then proposed,to provide fault detectability and fault isolability conditions, thus making possible fault tolerant control. Finally, we give an algorithm which allows to distribute a system into subsystems such that the problem of autonomous FDI is solvable for each of these subsystems.
\end{abstract}

Keywords: Networked Control Systems, Fault Diagnosis, Fault Tolerant Control, Decentralized Design, Structural analysis, Graph-theoretic approach.

\section{INTRODUCTION}

Networked Control systems (NCS) are in general composed of a large number of interconnected devices that exchange data through communication networks; examples include industrial automation, building supervision, automotive control, ... NCSs provide many advantages such as modular and flexible system design, fast implementation, distribution. But, some disadvantages such as loss of information, time delays in the data transmission may also have an effect on the control performances and should be taken into account in the design procedure. Since the early 70's, Fault Detection and Isolation (FDI) in dynamic system has received much attention from both theoretical and application viewpoints (Franck and Ding, 2000; Gertler, 1998; Chen and Patton, 1999). The fault tolerant control problem is a more recent research topic but there also various results on it (Zhang and Jiang, 2003). The task to be tackled in achieving fault-tolerance is the design of a controller with suitable structure to guarantee satisfactory performance, not only when all control components are operational, but also in the case when instruments are operating under a faulty mode. If we compare with the development in FDI/FTC for small scale centralized systems, corresponding research for large scale interconnected systems is very limited. This is in particular the case for the NCS's systems which are considered here, such as the example represented on Figure 1. The FDI/FTC system is distributed over the network, and decomposed into subsystems, each of which being in charge of a local FDI/FTC unit. The information required by each unit from the others being transmitted via the networks, it is therefore important for fault tolerance to consider the network faults which can occur. A second new problem concerns complex systems, controlled through a network of controllers, each one dedicated to a subset of components, and communicating with 
each others, because as the components are physically interconnected, the control system must take it into account. This is specifically important when a fault occurs, because this fault will propagate along the process, and isolated local diagnosis could lead to a wrong result. The theoretical problem is related to the distribution of diagnosis between several "diagnosers" and how to make them communicate (which information must they share? This study should take into account the plant physical structure, the components interactions, the algorithms complexity, etc. The aim of the paper is to study the global as well as the local fault detectability and isolabilty which represent the necessary conditions before a distributed implementation of a FDI/FTC system.

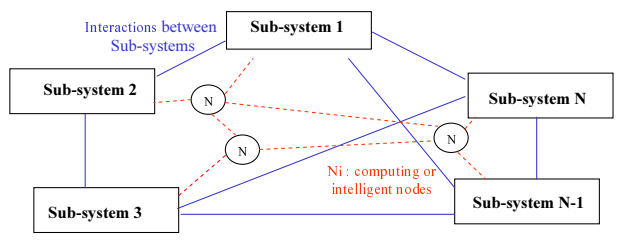

Figure 1. : Networked Control Systems

The paper is organized as follows. In the next section, we formulate general statements about different architectures for FDI/FTC: centralized, decentralized FDI, and autonomous distributed. The residual generation problem for FDI is addressed, considering designs based on the use of directional filters. The third section is dedicated to the structural analysis of each of the previous architectures. Using a graph-theoretic approach, we give necessary and sufficient conditions to the detectability and isolability of any chosen part of the fault vector for any of the three FDI/FTC schemes. Some concluding remarks end the paper.

\section{PROBLEM STATEMENT}

A classical way to achieve fault-tolerant control relies on supervised control where an FDI unit provides information about the location and time occurrence of any fault. Faults are compensated via an appropriate control law triggered according to diagnosis of the system (Noura et al., 2000). Once the FDI module indicates which sensor or actuator is faulty, the fault magnitude can be estimated and a new control law is added to the nominal one to thwart the fault effect on the system.

Generation of residuals having directional properties in response to a particular faults is an attractive way for enhancing fault isolability. A fault detection filter is a special dynamic observer which generates directional residuals. It was first developed by Beard (Beard, 1971) and Jones (Jones, 1973) and later revisited by (Massoumnia, 1986) in the geometric framework and by White and Speyer (White and Speyer, 1987) in the context of eigenstructure assignment. Further improvements were suggested by Park and Rizzoni (Park and Rizzoni, 1994) and Liu and Si (Liu and $\mathrm{Si}, 1997)$.

Let us suppose the system under study can be described by the following model :

$$
\left\{\begin{array}{l}
\dot{x}(t)=A x(t)+B u(t)+E f(t) \\
y(t)=C x(t)
\end{array}\right.
$$

where $x(t) \in \mathbb{R}^{n}$ is state vector, $y(t) \in \mathbb{R}^{m}$ the output vector, $u(t) \in \mathbb{R}^{p}$ the input vector. $E$ is faults distribution matrix and $f(t) \in \mathbb{R}^{q}$ is the fault vector. We also consider the observer:

$$
\dot{\hat{x}}(t)=A \hat{x}(t)+B u(t)+K(y(t)-C \hat{x}(t))
$$

and residual generator:

$$
r(t)=L C(x(t)-\hat{x}(t))
$$

The goal is to compute the gain $K$ such that transfer matrix $W(s)$ from $f$ to $r$ is given by:

$$
W(s)=L C(s I-(A-K C))^{-1} E=\left(\frac{1}{s}\right) I
$$

where (4) ensures the diagonal structure of transfer from faults to residuals allowing the multiple faults isolation. We have:

$$
\begin{aligned}
W(s) & =L C(s I-(A-K C))^{-1} E \\
& =\sum_{k \geq 0} s^{-k-1}\left[\ldots\left|L C(A-K C)^{k} E_{i}\right| \ldots\right]
\end{aligned}
$$

where $\sum_{k \geq 0} s^{-k-1} L C(A-K C)^{k} E_{i}=s^{-1} L C E_{i}+$ $s^{-2} L C(A-K C) E_{i}+s^{-3} L C(A-K C)^{2} E_{i}+\ldots$ Hence, if the observer's gain $K$ satisfies the algebraic constraint:

$$
(A-K C)\left[\ldots\left|E_{i}\right| \ldots\right]=(A-K C) E=0
$$

then (5) gives:

$$
W(s)=\left[\ldots\left|L C E_{i} s^{-1}\right| \ldots\right]=D\left(\frac{1}{s}\right) I
$$

with $D=L C E$ and (4) is satisfied under $D=I$.

Solution to this problem makes use of the following theorem by Keller (J.-Y. Keller, 1999).

Theorem 1. Under $\operatorname{rank}(D)=q$ which implies $q<m$, the solution of the eigenstructure assignment (6) can be parameterized according to

$$
K=\omega \Pi+\bar{K} \Sigma
$$

with $\Sigma=\beta(I-D \Pi), \Pi=D^{-1}$ and $\omega=A E$.

where $\beta \in \mathbb{R}^{m-q, m}$ is an arbitrary matrix determined so that matrix $\Sigma$ is of full rows rank and $\bar{K}$ is the reduced gain describing the remaining degree of freedom.

Considering theorem 1, we obtain :

$$
\dot{\hat{x}}(t)=A \hat{x}(t)+B u(t)+\omega q(t)+\bar{K} \gamma(t)
$$

Furthermore, it is easy to show that $\gamma(t)=$ $\Sigma(y(t)-C \hat{x}(t))$ is decoupled from the faults, while $q(t)=\Pi(y(t)-C \hat{x}(t))$ depends on the fault. Hence, it is possible to give a fault free estimate of the actual state vector according to:

$$
\bar{x}(t)=\left(\begin{array}{c}
\Sigma C \\
\Pi C
\end{array}\right)^{+}\left(\begin{array}{c}
\Sigma C \hat{x}(t) \\
\Pi C \hat{x}(t)+q(t)
\end{array}\right)
$$

With respect to eq. (7) an estimation of the faults can be obtained according to $\hat{f}(t)=\dot{q}(t)$. For FTC, the goal 
is to make the nominal system and the reconfigured system as closed as possible. Once the fault is isolated, the corresponding fault estimation and compensation module is switched on to reduce the fault effect on the system. The control signal applied to the system is given by

$$
u(t)=-G \bar{x}(t)+u_{a d}(t)
$$

and is computed such that the fault influence on to the system is compensated. In other terms, it must satisfy

$$
B u_{a d}(t)+E \hat{f}(t)=0
$$

the solution of (12) can be obtained if $\operatorname{rank}\left(\left[\begin{array}{ll}B & E\end{array}\right]\right)=$ $\operatorname{rank}(E)$.

In this classical centralized design, FTC can be represented by Fig. 2 which clearly shows that all the available inputs and outputs information are collected and processed in a central node.

The aim of the paper is to develop a distributed FDI

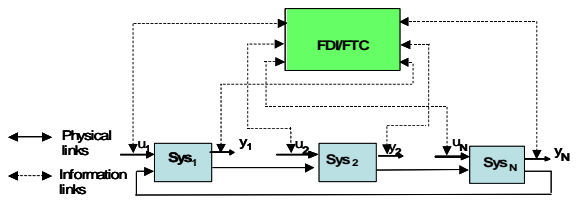

Figure 2. : Centralized architecture for FDI/FTC

architecture for networked control systems. A distributed FDI system is one that consists of several localized FDI sub-systems, acting on a single subsystem, in order to achieve a global FDI objective.

Consider the structured linear system $(\Sigma)(1)$. As such system is a distributed system, it is constituted of several subsystems $\left(\Sigma_{i}\right), i=1, \ldots, N$. Each subsystem satisfies to a model of the form:

$$
\left\{\begin{array}{l}
\dot{x}_{i}(t)=A_{i} x_{i}(t)+\sum_{j=1, j \neq i}^{N} H_{i, j} x_{j}(t)+B_{i} u_{i}(t)+E_{i} f_{i}(t) \\
y_{i}(t)=C_{i} x_{i}(t)
\end{array}\right.
$$

where for $i=1, \ldots, N, x_{i} \in \mathbb{R}^{n_{i}}$ is the state vector of subsystem $\left(\Sigma_{i}\right), y_{i} \in \mathbb{R}^{m_{i}}$ is the output vector of subsystem $\left(\Sigma_{i}\right), u_{i} \in \mathbb{R}^{p_{i}}$ is the output vector of subsystem $\left(\Sigma_{i}\right) f_{i} \in \mathbb{R}^{q_{i}}$ is the fault vector occurring on subsystem $\left(\Sigma_{i}\right)$.

Consider also the following decentralized residual generators for $i=1, \ldots, N$

$$
\left\{\begin{array}{l}
\dot{\hat{x}}_{i}(t)=A_{i} \hat{x}_{i}(t)+K_{i}\left(y_{i}(t)-C_{i} \hat{x}_{i}(t)\right)+\sum_{j=1, j \neq i}^{N} H_{i, j} \bar{x}_{j}(t)+B_{i} u_{i}(t) \\
r_{i}(t)=L_{i}\left(y_{i}(t)-C_{i} \hat{x}_{i}(t)\right)
\end{array}\right.
$$

where $\bar{x}_{j}(t)$ is a fault free state estimate, $r_{i}(t)$ the output of the filter and where $K_{i} \in \mathbb{R}^{n_{i}, m_{i}}$ are unknown matrices that will be designed in order to fulfill local fault detection and isolation requirements.

If we consider that :

$$
\lim _{t \rightarrow \infty} x_{i}(t)-\bar{x}_{i}(t)=0, \quad i=1, \ldots, N
$$

then the local estimation errors and residuals satisfy :

$$
\left\{\begin{array}{l}
\dot{\varepsilon}_{i}(t)=\left(A_{i}-K_{i} C_{i}\right) \varepsilon_{i}(t)+E_{i} f_{i}(t) \quad i=1, \ldots, N \\
r_{i}(t)=L_{i} C_{i} \varepsilon_{i}(t)
\end{array}\right.
$$

Thus, in order to deal with a decentralized design of the FDI/FTC approach, the following conditions should be satisfied :

$$
\begin{aligned}
& \lim _{t \rightarrow \infty}\left(f_{i}-\hat{f}_{i}\left(\hat{x}_{i}, u_{i}, y_{i}, \bar{x}_{j}\right)\right)=0 \\
& \lim _{t \rightarrow \infty}\left(x_{i}-\bar{x}_{i}\left(\hat{x}_{i}, u_{i}, y_{i}, \bar{x}_{j}\right)\right)=0 \forall f_{i}(t)
\end{aligned}
$$

In this decentralized design, FTC can be represented by Fig 3 . In the decentralized design, each local

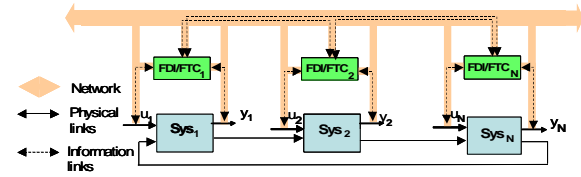

Figure 3. : Decentralized networked FDI

FDI/FTC unit requires information from the others. But when the network fails, these information are no longer available and the local FDI/FTC unit must be able to perform its work autonomously. Following the same idea as previously, a way to design a fully distributed autonomous FDI/FTC is to consider the following residual generator :

$$
\left\{\begin{array}{l}
\dot{\hat{x}}_{i}(t)=A_{i} \hat{x}_{i}(t)+K_{i}\left(y_{i}(t)-C_{i} \hat{x}_{i}(t)\right)+B_{i} u_{i}(t) \\
r_{i}(t)=L_{i}\left(y_{i}(t)-C_{i} \hat{x}_{i}(t)\right)
\end{array}\right.
$$

Fig. 4 gives an illustration of the distributed autonomous design. Finally, what is possible to do from

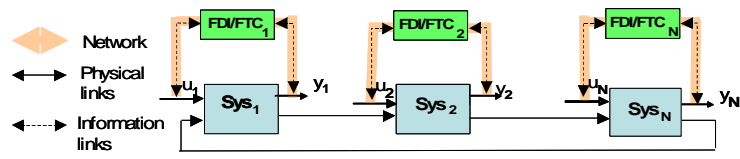

Figure 4. : Distributed autonomous FDI

the FDI/FTC point of views strongly depends on the fault detectability and isolabilty conditions.

\section{STRUCTURAL ANALYSIS OF THE FAULT DETECTABILITY AND ISOLABILITY FOR DISTRIBUTED SYSTEMS}

In this section, we give the necessary conditions for the solvability of the observer-based FDI for distributed systems in different configurations: centralized FDI, decentralized FDI and autonomous FDI. To do so, we use a graph theoretic approach, which is well-adapted to handle the structural properties of the studied systems and to deal with large scale systems. In many modeling problems, system matrices have a number of fixed zero entries determined by the physical structure while the remaining entries are not precisely known. To study the properties of these systems in spite of the poor knowledge we have on them, the idea is that we only keep the zero/nonzero entries in the state space matrices. Thus, we consider models where the fixed zeros are conserved while the 
nonzero entries are replaced by free parameters. There is a huge amount of interesting works using this kind of models called structured models since their study requires a low computational burden which allows to deal with large scale systems. Many studies on structured systems are related to the graph-theoretic approach. Survey paper (Dion et al., 2003) reviews the most significant results in this area. From these works, it results that the graph-theoretic approach provides simple and elegant solutions and so is very well suited to analyse large scale or/and uncertain systems.

\subsection{Graphical representation of a structured linear system for FDI purpose}

Consider structured linear system (1) which is constituted of several subsystems $\left(\Sigma_{i}\right), i=1, \ldots, N$ :

$\left(\Sigma_{i}\right)\left\{\begin{array}{l}\dot{x}_{i}(t)=A_{i} x_{i}(t)+B_{i} u_{i}(t)+\sum_{j=1, j \neq i}^{N} H_{i, j} x_{j}(t)+E_{i} f_{i}(t) \\ y_{i}(t)=C_{i} x_{i}(t)\end{array}\right.$

where for $i=1, \ldots, N, x_{i} \in \mathbb{R}^{n_{i}}$ is the state vector of subsystem $\left(\Sigma_{i}\right), y_{i} \in \mathbb{R}^{m_{i}}$ is the output vector of subsystem $\left(\Sigma_{i}\right), u_{i} \in \mathbb{R}^{p_{i}}$ is the output vector of subsystem $\left(\Sigma_{i}\right) f_{i} \in \mathbb{R}^{q_{i}}$ is the fault vector occurring on subsystem $\left(\Sigma_{i}\right) . A_{i}, B_{i}, H_{i, j}, E_{i}, C_{i}$ represent matrices of appropriate dimensions whose elements are either fixed to zero or assumed to be free non-zero parameters $\Lambda=\left(\lambda_{1}, \lambda_{2}, \ldots, \lambda_{h}\right)^{T}$. If all the non-zeros parameters $\lambda_{i}$ are fixed, we obtain an admissible realization of structured system $\left(\Sigma_{\Lambda}\right)$. We say that a property is true generically if it is true for almost all realizations of the system or for almost all parameter values. To structured system $\left(\Sigma_{i}\right)$, we associate a digraph noted $\mathcal{G}\left(\Sigma_{\Lambda}\right)$ which is constituted by a vertex set $\mathcal{V}$ and an edge set $\mathcal{E}$. More precisely, $\mathcal{V}=\bigcup_{i=1}^{N}\left(\mathbf{X}_{\mathbf{i}} \cup \mathbf{U}_{\mathbf{i}} \cup \mathbf{Y}_{\mathbf{i}} \cup \mathbf{F}_{\mathbf{i}}\right)$, where $\mathbf{X}_{\mathbf{i}}=\left\{\mathbf{x}_{\mathbf{i}, \mathbf{1}}, \ldots, \mathbf{x}_{\mathbf{i}, \mathbf{n}_{\mathbf{i}}}\right\}$ is the set of state vertices for subsystem $i, \mathbf{U}_{\mathbf{i}}=\left\{\mathbf{u}_{\mathbf{i}, \mathbf{1}}, \ldots, \mathbf{u}_{\mathbf{i}, \mathbf{p}_{\mathbf{i}}}\right\}$ is the set of input vertices for subsystem $i, \mathbf{Y}_{\mathbf{i}}=\left\{\mathbf{y}_{\mathbf{i}, \mathbf{1}}, \ldots, \mathbf{y}_{\mathbf{i}, \mathbf{m}_{\mathbf{i}}}\right\}$ is the set of output vertices for subsystem $i$ and $\mathbf{F}_{\mathbf{i}}=\left\{\mathbf{f}_{\mathbf{i}, \mathbf{1}}, \ldots, \mathbf{f}_{\left.\mathbf{i}, \mathbf{q}_{\mathbf{i}}\right\}}\right\}$ is the set of failure vertices for subsystem $i$. The edge set is

$\mathcal{E}=\bigcup_{i=1}^{N}\left(A_{i}\right.$-edges $\cup B_{i}$-edges $\cup C_{i}$-edges $\cup E_{i}$-edges $\cup$ $\bigcup_{j=1, j \neq i}^{N} H_{i, j}$-edges $)$, where

$A_{i}$-edges $\quad=\quad\left\{\left(\mathbf{x}_{\mathbf{i}, \mathbf{j}}, \mathbf{x}_{\mathbf{i}, \mathbf{k}}\right) \mid A_{i}(k, j) \neq 0\right\}$, $B_{i}$-edges $\quad=\quad\left\{\left(\mathbf{u}_{\mathbf{i}, \mathbf{h}}, \mathbf{x}_{\mathbf{i}, \mathbf{l}}\right) \mid B_{i}(l, h) \neq 0\right\}$, $H_{i, j}$-edges $\quad=\quad\left\{\left(\mathbf{x}_{\mathbf{j}, \mathbf{l}}, \mathbf{x}_{\mathbf{i}, \mathbf{h}}\right) \mid H_{i, j}(h, l) \neq 0\right\}$, $E_{i}$-edges $\quad=\quad\left\{\left(\mathbf{f}_{\mathbf{i}, \mathbf{h}}, \mathbf{x}_{\mathbf{i}, \mathbf{l}}\right) \mid E_{i}(l, h) \neq 0\right\} \quad$ and $C_{i}$-edges $=\left\{\left(\mathbf{x}_{\mathbf{i}, \mathbf{h}}, \mathbf{y}_{\mathbf{i}, \mathbf{l}}\right) \mid C_{i}(l, h) \neq 0\right\}$.

Here, $M(i, j)$ is the $(i, j)$ th element of matrix $M$ and $\left(\mathbf{v}_{\mathbf{1}}, \mathbf{v}_{\mathbf{2}}\right)$ denotes a directed edge from vertex $\mathbf{v}_{\mathbf{1}} \in \mathcal{V}$ to vertex $\mathbf{v}_{\mathbf{2}} \in \mathcal{V}$. The following example illustrates the previous settings.
Example 2. Consider the structured subsystem 1 of a distributed system constituted of three subsystems: $A_{1}=\left(\begin{array}{ccccccc}0 & 0 & 0 & 0 & 0 & 0 & 0 \\ 0 & 0 & 0 & 0 & 0 & 0 & 0 \\ \times & 0 & 0 & 0 & 0 & 0 & 0 \\ 0 & \times & 0 & 0 & 0 & 0 & 0 \\ 0 & 0 & 0 & 0 & 0 & 0 & 0 \\ 0 & 0 & \times & 0 & 0 & 0 & 0 \\ 0 & 0 & \times & \times & \times & 0 & 0\end{array}\right), B_{1}=\left(\begin{array}{c}\times \\ 0 \\ 0 \\ 0 \\ 0 \\ 0 \\ 0\end{array}\right), E_{1}=\left(\begin{array}{ccc}\times & \times & 0 \\ 0 & \times & 0 \\ 0 & 0 & 0 \\ 0 & 0 & 0 \\ 0 & 0 & \times \\ 0 & 0 & 0 \\ 0 & 0 & 0\end{array}\right)$ $C_{1}=\left(\begin{array}{ccccccc}0 & 0 & 0 & 0 & 0 & \times & 0 \\ 0 & 0 & 0 & 0 & 0 & \times & \times\end{array}\right), H_{1,2}=H_{1,3}=0, A_{2}=\left(\begin{array}{cccccc}0 & 0 & 0 & 0 & 0 & 0 \\ 0 & 0 & 0 & 0 & 0 & 0 \\ 0 & 0 & 0 & 0 & 0 & 0 \\ \times & \times & 0 & 0 & 0 & 0 \\ 0 & \times & 0 & 0 & 0 & 0 \\ 0 & 0 & 0 & 0 & 0 & 0\end{array}\right)$, $B_{2}=\left(\begin{array}{cc}\times & 0 \\ 0 & \times \\ 0 & 0 \\ 0 & 0 \\ 0 & 0 \\ 0 & 0\end{array}\right), C_{2}=\left(\begin{array}{cccccc}0 & 0 & \times & 0 & 0 & 0 \\ 0 & 0 & \times & \times & 0 & 0 \\ 0 & 0 & 0 & 0 & \times & \times\end{array}\right), E_{2}=\left(\begin{array}{cc}0 & 0 \\ \times & 0 \\ 0 & 0 \\ 0 & 0 \\ 0 & 0 \\ 0 & \times\end{array}\right), H_{2,1}=$ $\left(\begin{array}{ccccccc}0 & 0 & 0 & 0 & 0 & 0 & 0 \\ 0 & 0 & 0 & 0 & 0 & 0 & 0 \\ 0 & 0 & 0 & 0 & \times & 0 & 0 \\ 0 & 0 & 0 & 0 & \times & 0 & 0 \\ 0 & 0 & 0 & 0 & 0 & 0 & 0 \\ 0 & 0 & 0 & 0 & 0 & 0 & 0\end{array}\right), H_{2,3}=0, A_{3}=\left(\begin{array}{cccccc}0 & 0 & 0 & 0 & 0 & 0 \\ \times & 0 & 0 & 0 & 0 & 0 \\ \times & 0 & 0 & 0 & 0 & 0 \\ 0 & \times & 0 & 0 & 0 & 0 \\ 0 & 0 & \times & 0 & 0 & 0 \\ 0 & 0 & 0 & 0 & 0 & \times\end{array}\right), B_{3}=\left(\begin{array}{ccc}\times & 0 & 0 \\ 0 & 0 & 0 \\ \times & \times & 0 \\ 0 & 0 & 0 \\ 0 & 0 & 0 \\ 0 & 0 & \times\end{array}\right)$ $E=\left(\begin{array}{c}\times \\ 0 \\ 0 \\ 0 \\ 0 \\ 0\end{array}\right) H_{3,1}=\left(\begin{array}{lllllll}0 & 0 & 0 & 0 & 0 & 0 & 0 \\ 0 & 0 & 0 & 0 & 0 & 0 & 0 \\ 0 & 0 & 0 & \times & 0 & 0 & 0 \\ 0 & 0 & 0 & 0 & 0 & 0 & 0 \\ 0 & 0 & 0 & 0 & 0 & 0 & 0 \\ 0 & 0 & 0 & 0 & 0 & 0 & 0\end{array}\right), H_{3,2}=\left(\begin{array}{llllll}0 & 0 & 0 & 0 & 0 & 0 \\ 0 & \times & 0 & 0 & 0 & 0 \\ 0 & 0 & 0 & 0 & 0 & 0 \\ 0 & 0 & 0 & 0 & 0 & 0 \\ 0 & 0 & 0 & 0 & 0 & 0 \\ 0 & 0 & 0 & 0 & 0 & 0\end{array}\right)$ and $C_{3}=\left(\begin{array}{cccccc}0 & 0 & 0 & \times & 0 & 0 \\ 0 & 0 & 0 & 0 & \times & \times\end{array}\right)$. Here, for the sake of simplicity, we represent each nonzero parameter $\lambda_{i}$ of the structured system by the symbol $\times$. To such a model, we associate the digraph in figure 5 .

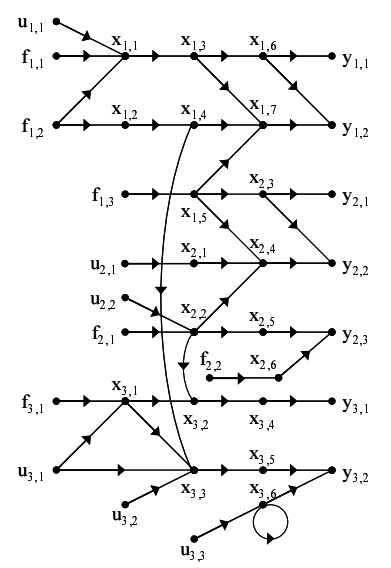

Figure 5. Digraph associated to Example 2

We denote path $\mathbf{P}$ containing vertices $\mathbf{s}_{\mathbf{r}_{0}}, \mathbf{s}_{\mathbf{r}_{1}}, \ldots, \mathbf{s}_{\mathbf{r}_{\mathbf{i}}}$ by $\mathbf{P}=\mathbf{s}_{\mathbf{r}_{0}} \rightarrow \mathbf{s}_{\mathbf{r}_{1}} \rightarrow \ldots \rightarrow \mathbf{s}_{\mathbf{r}_{\mathbf{i}}}$ where $\left(\mathbf{s}_{\mathbf{r}_{\mathbf{j}}}, \mathbf{s}_{\mathbf{r}_{\mathbf{j}+1}}\right) \in \mathcal{E}$ for $j=0,1 \ldots, i-1$. Some paths are disjoint if they have no common vertex. In the sequel, $\mathbf{V}_{\mathbf{1}}$ and $\mathbf{V}_{\mathbf{2}}$ denote two subsets of $\mathcal{V}$. A path $\mathbf{P}$ is said a $\mathbf{V}_{\mathbf{1}}-\mathbf{V}_{\mathbf{2}}$ path if its begin vertex belongs to $\mathbf{V}_{\mathbf{1}}$ and its end vertex belongs to $\mathbf{V}_{\mathbf{2}}$. We denote by $\rho\left[\mathbf{V}_{\mathbf{1}}, \mathbf{V}_{\mathbf{2}}\right]$ the maximal number of disjoint $\mathbf{V}_{\mathbf{1}}-\mathbf{V}_{\mathbf{2}}$ paths.

\subsection{Centralized FDI schemes}

The necessary and sufficient conditions for the solvability of the observer-based FDI problem are directly deduced from (Commault et al., 2002).

Proposition 3. Consider structured system $\left(\Sigma_{\Lambda}\right)$ represented by digraph $\mathcal{G}\left(\Sigma_{\Lambda}\right)$. The fault components asso- 
ciated to any $\overline{\mathbf{F}} \subseteq \mathbf{F}$ are isolable and detectable using a centralized observer-based FDI scheme iff in $\mathcal{G}\left(\Sigma_{\Lambda}\right)$

$$
\rho[\mathbf{F}, \mathbf{Y}]=\operatorname{card}(\overline{\mathbf{F}})+\rho[\mathbf{F} \backslash \overline{\mathbf{F}}, \mathbf{Y}]
$$

In example 2 , since $\rho[\mathbf{F}, \mathbf{Y}]=6$ and $\operatorname{card}(\mathbf{F})=6$, all the faults are detectable and isolable.

\subsection{Decentralized FDI scheme}

Assume now that, for some $j \in J \subseteq$ $\{1,2, \ldots, i-1, i+1, \ldots, N\}$, all the magnitude of measurement $Y_{j}$ and input $U_{j}$ are transmitted via the network to subsystem $\left(\Sigma_{i}\right)$.

Proposition 4. Consider structured system $\left(\Sigma_{\Lambda}\right)$ represented by digraph $\mathcal{G}(\Sigma)$. For subsystem $i$, the fault components associated to any $\overline{\mathbf{F}} \subseteq \mathbf{F}$ are generically detectable and isolable using a decentralized observerbased FDI scheme if and only if

$$
\begin{aligned}
& \rho\left[\left(\bigcup_{j \notin J \cup\{i\}} \mathbf{U}_{\mathbf{j}}\right) \cup \mathbf{F}, \bigcup_{j \in J \cup\{i\}} \mathbf{Y}_{\mathbf{j}}\right]= \\
& \quad \operatorname{card}(\overline{\mathbf{F}})+\rho\left[\left(\bigcup_{j \notin J \cup\{i\}} \mathbf{U}_{\mathbf{j}}\right) \cup(\mathbf{F} \backslash \overline{\mathbf{F}}), \bigcup_{j \in J \cup\{i\}} \mathbf{Y}_{\mathbf{j}}\right]
\end{aligned}
$$

\subsection{Decentralized autonomous FDI scheme}

Now, we consider that no information from subsystems $\left(\Sigma_{j}\right), j \neq i$ arrive to subsystem $\left(\Sigma_{i}\right)$.

Proposition 5. Consider structured system $\left(\Sigma_{\Lambda}\right)$ represented by digraph $\mathcal{G}(\Sigma)$. For subsystem $i$, the fault components associated to any $\overline{\mathbf{F}} \subseteq \mathbf{F}_{\mathbf{i}}$ are detectable and isolable using a decentralized observer-based FDI scheme iff

$$
\rho\left[\left(\bigcup_{j \neq\{i\}} \mathbf{U}_{\mathbf{j}}\right) \cup \mathbf{F}, \mathbf{Y}_{\mathbf{i}}\right]=\operatorname{card}(\overline{\mathbf{F}})+\rho\left[\left(\bigcup_{j \neq\{i\}} \mathbf{U}_{\mathbf{j}}\right) \cup(\mathbf{F} \backslash \overline{\mathbf{F}}), \mathbf{Y}_{\mathbf{i}}\right]
$$

Considering example 2, we can do the following computations:

- Subsystem 1:

for $J=\{2\}$ or for $J=\{3\}: \forall \overline{\mathbf{F}} \subseteq \mathbf{F}_{\mathbf{1}}$,
$\rho\left[\left(\bigcup_{j \notin J \cup\{i\}} \mathbf{U}_{\mathbf{j}}\right) \cup \mathbf{F}, \bigcup_{j \in J \cup\{i\}} \mathbf{Y}_{\mathbf{j}}\right]+\operatorname{card}(\overline{\mathbf{F}})+$
$\rho\left[\left(\bigcup_{j \notin J \cup\{i\}} \mathbf{U}_{\mathbf{j}}\right) \cup(\mathbf{F} \backslash \overline{\mathbf{F}}), \bigcup_{j \in J \cup\{i\}} \mathbf{Y}_{\mathbf{j}}\right]$. for $J=\{2,3\}$, condition (18) is satisfied for every $\overline{\mathbf{F}} \subseteq \mathbf{F}_{\mathbf{1}}$. So the faults of subsystem 1 can be detectable and isolable using distributed FDI scheme only since we consider that all the inputs and output measurement of subsystems 2 and 3 are transmitted via the network to subsystem 1 .

- Subsystem 2: $\rho\left[\bigcup_{j=1,2} \mathbf{U}_{\mathbf{j}} \cup \mathbf{F}, \mathbf{Y}_{\mathbf{2}}\right]=3$ and $\rho\left[\bigcup_{j=1,2} \mathbf{U}_{\mathbf{j}} \cup \mathbf{F} \backslash \mathbf{F}_{\mathbf{2}}, \mathbf{Y}_{\mathbf{2}}\right]=1$. Consequently, for this subsystem, all the faults are generically detectable and isolable using an autonomous decentralized observer-based FDI scheme.
- Subsystem 3: $f_{3}$ is generically detectable and isolable using a decentralized observer-based FDI scheme only if the input and output measurements of subsystem 2 are transmitted to subsystem 3 .

\section{DISTRIBUTION OF FDI NODES}

In this last paragraph, using the previous results, the objective is to determine a distribution of the FDI task on local nodes such that the problem of autonomous local observer based FDI problem is generically solvable for each of these nodes.

Consider a global linear system (1), where $A, B, C$ and $E$ are structured matrices of appropriate dimensions. The objective is not exactly to obtain a subdivision of the global system into subsystems but to obtain a certain number of Control/FDI nodes such that each of them is able locally to detect and isolate a part of the faults occurring on the system.

As previously, we define a global digraph associated to system $\left(\Sigma_{\Lambda}\right)$ noted $\mathcal{G}_{g}\left(\Sigma_{\Lambda}\right)$. It is constituted by a vertex set $\mathcal{V}_{g}$ and an edge set $\mathcal{E}_{g}$ i.e. $\mathcal{G}\left(\Sigma_{\Lambda}\right)=\left(\mathcal{V}_{g}, \mathcal{E}_{g}\right)$. More precisely, $\mathcal{V}_{g}=\mathbf{X} \cup \mathbf{U} \cup \mathbf{Y} \cup \mathbf{F}$ and the edge set is $\mathcal{E}_{g}=A$-edges $\cup B$-edges $\cup E$-edges $\cup C$-edges. $\mathrm{We}$ define for each $\mathbf{V}_{\mathbf{Y}} \subseteq \mathbf{Y}$ functions $\alpha$ as:

$$
\begin{aligned}
\alpha: 2^{\mathbf{Y}} & \rightarrow 2^{\mathbf{F}} \\
\mathbf{V}_{\mathbf{Y}} & \mapsto \alpha\left(\mathbf{V}_{\mathbf{Y}}\right)=\left\{\mathbf{f}_{\mathbf{i}} \in \mathbf{F}, \text { such that } \rho\left[\mathbf{f}_{\mathbf{i}}, \mathbf{V}_{\mathbf{Y}}\right] \neq 0\right\}
\end{aligned}
$$

The digraph $\mathcal{G}_{g}\left(\Sigma_{\Lambda}\right)$ associated to the system of example 2 is depicted in figure 6 Note that the distribution

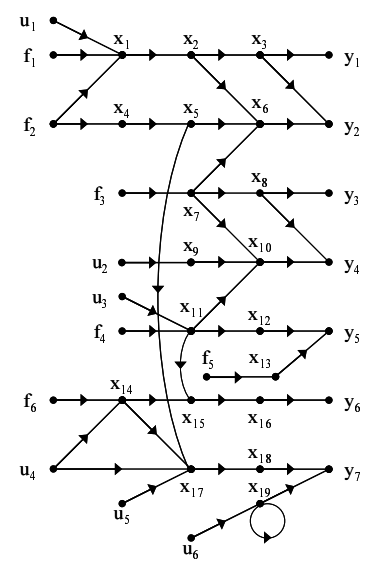

Figure 6. Digraph associated to Example 2

of the FDI nodes has a solution only if the observerbased FDI problem is solvable for the centralized scheme. Therefore, we assume that $\rho[\mathbf{F}, \mathbf{Y}]=\operatorname{card}(\mathbf{F})$. From Proposition 5, a subsystem defined by vertex subsets $\mathbf{V}_{\mathbf{Y}} \subseteq \mathbf{Y}$ and $\mathbf{V}_{\mathbf{U}} \subseteq \mathbf{U}$ is autonomous in a FDI context iff

$\rho\left[\mathbf{F} \cup\left(\mathbf{U} \backslash \mathbf{V}_{\mathbf{U}}\right), \mathbf{V}_{\mathbf{Y}}\right]=\operatorname{card}\left(\alpha\left(\mathbf{V}_{\mathbf{Y}}\right)\right)+\rho\left[\mathbf{U} \backslash \mathbf{V}_{\mathbf{U}}, \mathbf{V}_{\mathbf{Y}}\right]$

To establish this equality, we assume that the FDI node defined by the pair $\left(\mathbf{V}_{\mathbf{U}}, \mathbf{V}_{\mathbf{Y}}\right)$ must detect and isolate all the fault components included in $\mathbf{V}_{\mathbf{Y}}$. Moreover, the subdivision of a subsystem defined by vertex subsets $\mathbf{V}_{\mathbf{Y}} \subseteq \mathbf{Y}$ and $\mathbf{V}_{\mathbf{U}} \subseteq \mathbf{U}$ is acceptable only if the rest of the system is also autonomous in a FDI context 
$\rho\left[\mathbf{F} \cup \mathbf{V}_{\mathbf{U}}, \mathbf{Y} \backslash \mathbf{V}_{\mathbf{Y}}\right]=\operatorname{card}\left(\alpha\left(\mathbf{Y} \backslash \mathbf{V}_{\mathbf{Y}}\right)\right)+\rho\left[\mathbf{V}_{\mathbf{U}}, \mathbf{Y} \backslash \mathbf{V}_{\mathbf{Y}}\right]$

We give hereafter an algorithm which allows to compute the set of vertex subsets $\Delta \subseteq 2^{\mathrm{U} \cup \mathbf{Y}}$.

Algorithm 1.

Initialisation: $\mathbf{Z}=\mathbf{U} \cup \mathbf{Y}, \Delta=\emptyset$.

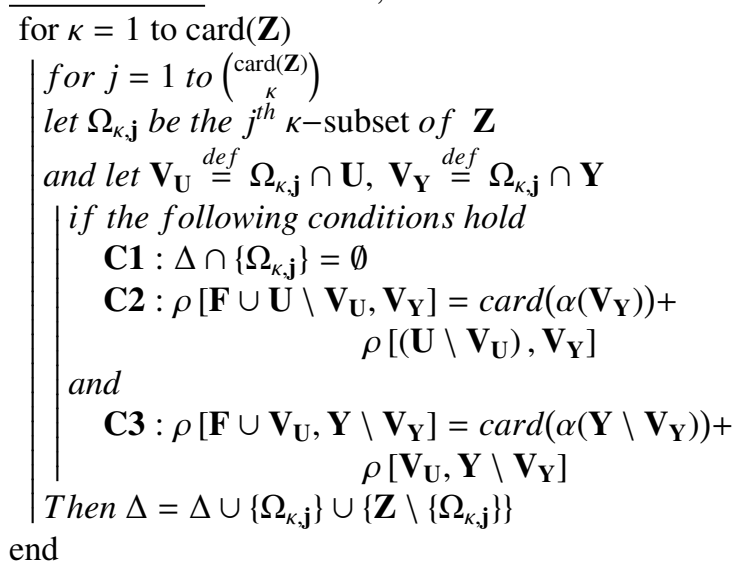

Proposition 6. Consider structured linear system $\left(\Sigma_{\Lambda}\right)$ represented by digraph $\mathcal{G}_{\}}\left(\Sigma_{\Lambda}\right)$. Consider a subdivision of the system into $N$ subsystems defined by the pairs of vertex subsets $\left(\mathbf{U}_{\mathbf{i}}, \mathbf{Y}_{\mathbf{i}}\right), i=1, \ldots, N$. The problem of autonomous observer based FDI problem is generically solvable for each of these subsystems iff:

- $\bigcup_{i=1}^{N} \mathbf{U}_{\mathbf{i}}=\mathbf{U}, \bigcup_{i=1}^{N} \mathbf{Y}_{\mathbf{i}}=\mathbf{Y}$,

- $\forall i \in\{1, \ldots, N\}$ and $\forall j \in\{1, \ldots, i\}$, we must have

$\mathbf{U}_{\mathbf{i}} \cap \mathbf{U}_{\mathbf{j}}=\emptyset$ and $\mathbf{Y}_{\mathbf{i}} \cap \mathbf{Y}_{\mathbf{j}}=\emptyset$,

- $\forall i \in\{1, \ldots, N\}, \mathbf{U}_{\mathbf{i}} \cup \mathbf{Y}_{\mathbf{i}} \in \Delta$.

In the case of the system defined in example (2), Algorithm 1 allows to compute set of vertex subsets

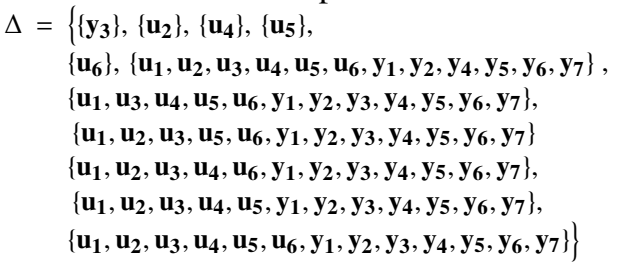

This set makes an inventory of all the possibilities for distributing the control/FDI nodes. The algorithm described above is not optimized and the computational point of view is not treated in this paper.

\section{CONCLUSION}

An important problem that must be considered when dealing with control over network, is the distribution of the FDI and FTC algorithms. An alternative approach to the classical centralized design for FDI/FTC has been studied in this paper. For that purpose, a residual generator with fixed directional properties has been considered. We have shown, that under some conditions, it is possible to distribute among several local processing units the residual generator and the resulting fault compensation mechanism. The fault detectability and isolability conditions for each configuration (centralized, distributed and autonomous NCS) have also been studied, considering a structural analysis approach and an algorithm which allows us to distribute the FDI task on local autonomous nodes is provided. In future works, information delays will be also considered in the design.

\section{REFERENCES}

Beard, R.V. (1971). Failure accommodation in linear systems through self-reorganization. Thesis. Massachusetts Institute of Technology. Department of Aeronautics and Astronautics. Cambridge, Massachusetts (USA).

Chen, J. and R.J. Patton (1999). Robust model-based fault diagnosis for dynamic systems. Vol. 3 of Kluwer international series on Asian studies in computer and information science. Kluwer Academic Publishers. Boston, U.S.A.

Commault, C., J-M. Dion, O. Sename and R. Motyeian (2002). Observer-based fault diagnosis for structured systems. IEEE Transactions on Automatic Control 47(12), 2074-2079.

Dion, J-M., C. Commault and J. W. van der Woude (2003). Generic properties and control of linear structured systems: A survey. Automatica 39(7), 1125-1144.

Franck, P. M. and S. Ding (2000). Development in the theory of FDI. In: IFAC Safeprocess Conference. Budapest, Hungary. pp. 16-27.

Gertler, J.J. (1998). Fault detection and diagnosis in engineering systems. Marcel Dekker. New York, U.S.A.

J.-Y. Keller (1999). Fault isolation filter design for linear stochastic systems. Automatica 35, 17011706.

Jones, H.L. (1973). Failure detection in linear systems. Thesis. Massachusetts Institute of Technology. Department of Aeronautics and Astronautics. Cambridge, Massachusetts (USA).

Liu, B. and J. Si (1997). Fault isolation filter design for linear time-invariant systems. IEEE Transactions on Automatic Control 42(5), 704-707.

Massoumnia, M. A. (1986). A geometric approach to the synthesis of failure detection filters. IEEE Transactions on Automatic Control 31(9), 839846.

Noura, H., D. Sauter, F. Hamelin and D. Theilliol (2000). Fault-tolerant control in dynamic systems : application to a winding machine. IEEE Control Systems Magazine 20(1), 33-49.

Park, J. and G. Rizzoni (1994). An eigenstructure assignment algorithm for the design of fault detection filters. IEEE Transactions on Automatic Control 39(7), 1521 - 1524.

White, J.E. and J.L. Speyer (1987). Detection filter design: spectral theory and algorithms. IEEE Transactions on Automatic Control 32(7), 593-603.

Zhang, Y. and J. Jiang (2003). Bibliographical review of reconfigurable fault tolerant control systems. In: IFAC Safeprocess Conference. Washington D.C., U.S.A. 